\title{
Effect of Laplacian of Gaussian Filter on Watermark Retrieval in Spatial domain Watermarking
}

\author{
Vahid Saffari ${ }^{*}$, Amirsoheil Ghazimoradi ${ }^{2}$ and Mehdi Alirezanejad ${ }^{3}$ \\ 'Department of Computer, College of Engineering, Shiraz Branch, Islamic Azad University, Shiraz, Iran; \\ Saffari@iaushiraz.ac.ir \\ 2Department of Computer, Babol Branch, Islamic Azad University, Babol, Iran; amirsoheilghazimoradi@yahoo.com \\ 32Department of Computer, Firoozkooh Branch, Islamic Azad University, Firoozkooh , Iran; m_arn2004@yahoo.com
}

\begin{abstract}
Laplacian of Gaussian filter (LOG) is an enhancement filter with less sensitivity to noise that is used for sharpening an image. The watermark is added as a noise to the host image in watermarking. The principal objective of this paper is to enumerate the beneficial effects of the LOG filter on watermark retrieval in spatial domain watermarking. This filter is applied before the extraction algorithm on the watermarked image which might be attacked. The distinction between the watermark and original image is increased by this filter. Thus, the probability of the watermark detection is augmented by it. Several image processing attacks is done to evaluate the effect of this filter on correlation based spatial domain watermarking. The effect of this filter on watermark recovery is statistically significant. The detection rate in noise addition and blurring is significant in compare with the similar method. However, the improvement in detection rate is also better in other common image processing attacks.
\end{abstract}

Keywords: Correlation Based Watermarking, Digital Image Watermarking, Laplacian of Gaussian, Spatial Domain Watermarking

\section{Introduction}

In recent years, application of digital watermarking in copyright protection is proved. In watermarking, some information is embedded invisibly in the host digital data $^{1,2}$.

There are two main watermarking methods category: the spatial domain or the transform domain ${ }^{3,4}$. The spatial domain is based on embedding the watermark directly in the signal of the host data. Generally, these methods are not robust against common image processing attack ${ }^{5,6}$.

Transform domain watermarking schemes increase the robustness of watermark by using properties of the transform. Generally, these methods are based on the Discrete Cosine Transform (DCT) ${ }^{7,8}$ and the Discrete Wavelet Transform (DWT) ${ }^{9,10}$.

The goal of the first group is to find most suitable place for watermark embedding. The characteristics of in frequency domain are utilized to detect proper places.
Methods such as Human Visual System (HVS) ${ }^{11,12}$, Just Noticeable Difference (JND) $)^{13,14}$ and optimal quantization matrix are the most applicable techniques for these purpose $^{15}$.

The purpose of the second group is to increase the robustness by augmenting the recovery algorithm. These methods intend to bold the watermark information for the recovery algorithms. The benefit of noise magnifying is taken in frequency domain and global minimum method to increase the detection rate ${ }^{16}$. The combination of Laplacian and Laplacian of Gaussian filtering is used to augment watermark detection rate in DCT based watermarking methods ${ }^{4,5}$. Alirezanejad et al. ${ }^{17,18}$ prove the effect of high boost filter on watermark recovery. It is applied before and within the watermark recovery algorithm to improve the extraction rate.

In this paper, application of Laplacian of Gaussian filter to augment the spatial domain algorithm is proposed. This filter is applied before the recovery algorithm. Different

${ }^{*}$ Author for correspondence 
experiments are done to show the impact of convolving Laplacian of Gaussian filter in the spatial domain based watermarking. This method and experimental results are explained in the following paragraphs.

\section{Materials And Methods}

\subsection{Laplacian of Gaussian Filter}

Laplacian filter is susceptible to noise ${ }^{19}$. To decrease the noise effect, Gaussian filter could be used. Thus, in Laplacian of Gaussian (LOG) operator, at first the Gaussian smoothing is applied, then the Laplacian operation is performed. The high frequency noise components could decrease by this combination. The sensibility to noise is decrease by using the combination of Gaussian function and Laplacian mask ${ }^{20}$. The LOG function for convolution is defined by the following Equation 1.

$$
\operatorname{LOG}(x, y)=\frac{1}{\pi \sigma^{4}}\left[1-\frac{x^{2}+y^{2}}{2 \sigma^{2}}\right] e^{\frac{x^{2}+y^{2}}{2 \sigma^{2}}}
$$

Where $\mathrm{x}$ and $\mathrm{y}$ are the corresponding row and column of the image, and $\sigma$ is standard deviation. LOG operator calculates the second spatial derivative of an image. The two-dimensional cross section of the zero-crossing of the Mexican hat is shown in Figure 1.

To utilize the Laplacian of Gaussian filter, at first the watermarked image is filtered by LOG, and then this image is subtracted from the watermarked image. Finally, the amplified value of it is subtracted from the LOG filtered image. This process is shown in the Figure 2.

\subsection{Typical Spatial Domain Watermarking}

A correlation based watermarking methods is used to show the effect of Laplacian filter on correlation based watermarking. In this paper, a typical correlation is used.

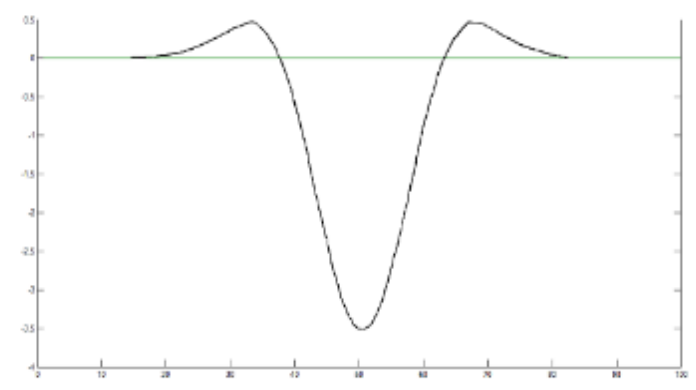

Figure 1. Cross section showing zero crossing.

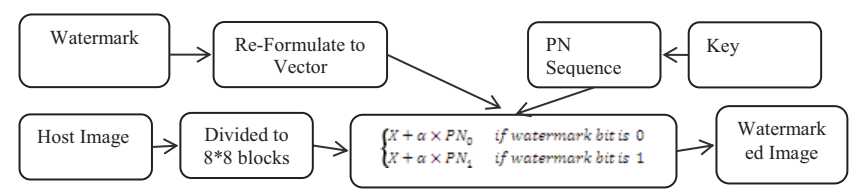

(a)

Figure 2. The proposed approach for utilizing the Laplacian of Gaussian filter.

This method is described by Alirezanejad et al. ${ }^{18}$. The embedding and extraction algorithm is shown in the Figure 3.

\section{Experimental Results}

Two standard grayscale images with size of $512 \times 512$ are used in our experiments, as shown in Figure 4(a)-(b). Peppers is used as a sample of with low spatial frequency image and Lena as a sample of with average spatial frequency image. In this experiment, a $49 \times 83$ binary image, as shown in Figure 4(c), is taken as the watermark of images. The robustness and imperceptibility of the proposed method is evaluated by applying the common signal processing operations. Peak Signal-to-Noise Ratio (PSNR), is employed to evaluate the imperceptible between an original image and watermarked image. The Normalized Absolute Difference (NAD) measure is used to evaluate the difference between an original watermark and the corresponding extracted one. If the method has lower NAD, it is more robust. The PSNR and the NAD are, respectively, defined by Equation 2 and 3; respectively,

$$
\begin{gathered}
\operatorname{PSNR}(\mathrm{O}, \overline{\mathrm{O}})=10 \log _{10} \frac{255 \times 255}{\sum_{\mathrm{i}=0 \mathrm{j}=0}^{\mathrm{I}-1 \mathrm{~J}-1}\left(\left\|\mathrm{O}_{\mathrm{ij}}-\overline{\mathrm{O}}_{\mathrm{ij}}\right\|\right)^{2}} \\
\operatorname{NAD}(\mathrm{W}, \overline{\mathrm{W}})=\frac{\sum_{i=0}^{M-1} \sum_{j=0}^{N-1}\left|W_{i, j}-\bar{W}_{i, j}\right|}{\sum_{i=0}^{M-1} \sum_{j=0}^{N-1}|W|}
\end{gathered}
$$

Where $\|\cdot\|_{1}$ and $|\cdot|$ stand for the L1 norm, and the number of components of a vector, respectively.

The watermark information is embedded with PSNR 35 , and $42 \mathrm{~dB}$ in the watermarked images. NAD between the original $\mathrm{W}$ and the extracted watermark $\mathrm{W}^{\prime}$ is calculated for different PSNRs. Experimental image processing 


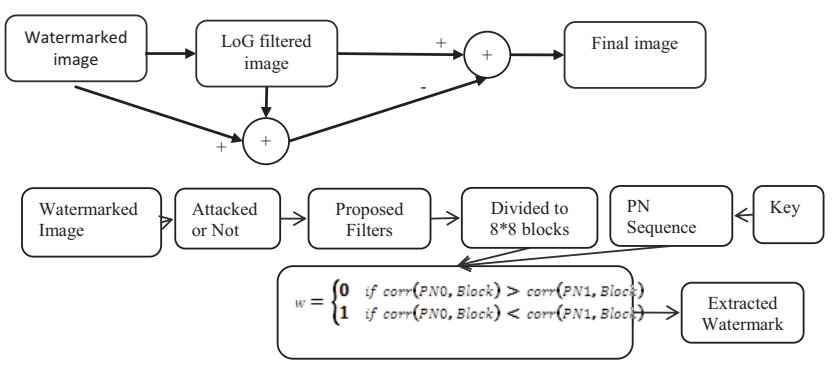

(b)

Figure 3. Correlation based watermarking algorithm: (a) Embedding algorithm, (b) Extraction algorithm.

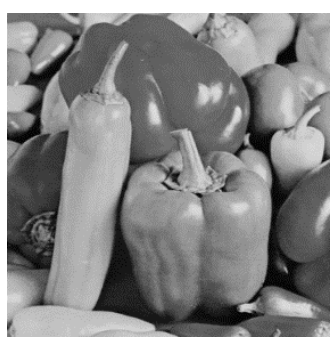

(a)

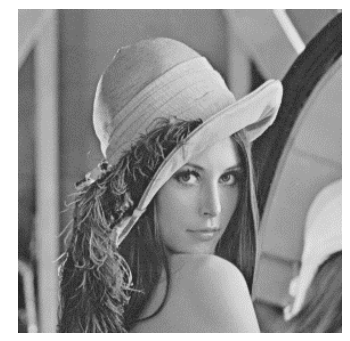

(b)

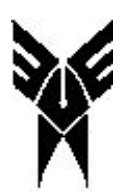

(c)
Figure 4. (a) The original Pepper image; (b) The original Lena image; (c) The original watermark.

operation are: 70\% JPEG compression, 65\% Image scaling, $10 \%$ adding Salt and Pepper noise, Gaussian filtering with $5 \times 5$ filter size, and Sigma equal to 1 . The presented method is implemented using MATLAB. LOG is implemented with MATLAB special command with $\mathrm{h}=5, \sigma=49$.

The Table 1 shows the value of NAD which is extracted from Peppers watermarked image. The extracted values obviously prove the efficiency of proposed method. In the attack free case, NAD value is decreased about 0.09 , and 0.13 for the 35 and $42 \mathrm{db}$ watermarked images, respectively. In JPEG compression this value is decreased about 0.15 and 0.19 , respectively. The improvement in the case of noise addition and blurring attack is more significant than the other attack. The NAD value is decreased about 0.42 for the noise addition and about 0.33 in the blurring attacks. In these attacks NAD values are three to six time better than the normal extraction. Although, in JPEG compression and scaling attack, NAD value improved two time better than before. Finally, it could conclude from the Table 1, that the improvement achieved by the proposed method for the low frequency image is significant.

The Table 2 shows the value of NAD which is extracted from Lena watermarked image. The efficiency of proposed method is proved by the extracted value. In the attack free
Table 1. The experimental results in term of NAD for Peppers watermarked image with different PSNR

\begin{tabular}{|c|c|c|c|}
\hline \multicolumn{2}{|c|}{ Attack } & & \multicolumn{2}{c|}{$\begin{array}{c}\text { Classical } \\
\text { Extraction }\end{array}$} & $\begin{array}{c}\text { Proposed } \\
\text { Extraction }\end{array}$ \\
\hline \multirow{2}{*}{ Attack Free } & 35 & 0.1047 & $\mathbf{0 . 0 1 9 8}$ \\
\cline { 2 - 4 } & 42 & 0.1971 & $\mathbf{0 . 0 6 0 3}$ \\
\hline JPEG Compression & 35 & 0.2162 & $\mathbf{0 . 0 6 5 9}$ \\
\cline { 2 - 4 }$(\mathbf{7 0 \% )}$ & 42 & 0.4541 & $\mathbf{0 . 2 6 9 1}$ \\
\hline \multirow{2}{*}{$\begin{array}{c}\text { Scaling (65\%) } \\
\text { Salt and Peppers } \\
\text { Noise Addition } \\
(\mathbf{1 0 \% )}\end{array}$} & 35 & 0.3440 & $\mathbf{0 . 1 4 4 5}$ \\
\cline { 2 - 4 } & 42 & 0.5028 & $\mathbf{0 . 2 5 3 2}$ \\
\hline \multirow{2}{*}{$\begin{array}{c}\text { Gaussian Blurring } \\
(H=5,6-1)\end{array}$} & 35 & 0.4979 & $\mathbf{0 . 0 7 8 8}$ \\
\cline { 2 - 4 } & 42 & 0.5678 & $\mathbf{0 . 2 4 3 4}$ \\
\hline
\end{tabular}

Table 2. The experimental results in term of NAD for Lena watermarked image with different PSNR

\begin{tabular}{|c|c|c|c|}
\hline & & \multicolumn{2}{|c|}{ NAD } \\
\hline Attack & & $\begin{array}{l}\text { Classical } \\
\text { Extraction }\end{array}$ & $\begin{array}{l}\text { Proposed } \\
\text { Extraction }\end{array}$ \\
\hline \multirow{2}{*}{ Attack Free } & 35 & 0.0600 & 0.0148 \\
\hline & 42 & 0.1445 & 0.0368 \\
\hline \multirow{2}{*}{$\begin{array}{c}\text { JPEG Compression } \\
(70 \%)\end{array}$} & 35 & 0.1635 & 0.0445 \\
\hline & 42 & 0.4093 & 0.2618 \\
\hline \multirow{2}{*}{ Scaling (65\%) } & 35 & 0.2888 & 0.1233 \\
\hline & 42 & 0.4193 & 0.2174 \\
\hline \multirow{2}{*}{$\begin{array}{l}\text { Salt and Peppers Noise } \\
\text { Addition }(10 \%)\end{array}$} & 35 & 0.4712 & 0.0735 \\
\hline & 42 & 0.6389 & 0.2140 \\
\hline \multirow{2}{*}{$\begin{array}{l}\text { Gaussian Blurring } \\
\qquad(\mathrm{H}=5, \sigma=1)\end{array}$} & 35 & 0.3428 & 0.1067 \\
\hline & 42 & 0.4739 & 0.1989 \\
\hline
\end{tabular}

case, NAD value is decreased about 0.05 , and 0.11 for the 35 and $42 \mathrm{db}$ watermarked images, respectively. In JPEG compression this value is decreased about 0.12 and 0.15 , respectively. In the scaling attack this value is decreased about 0.16 and 0.20 , respectively, the improvement in the case of noise addition and blurring attack is more significant than the other attack. The NAD value is decreased about 0.40 for the noise addition and about 0.25 in the blurring attacks. In these attacks NAD values are three to five time better than the normal extraction. Although, in JPEG compression and scaling attacks, NAD value improved 
two time better than before. It could be determine from the Table 2 , that the improvement attained by the proposed method for the mid frequency image is significant.

\section{Conclusion}

In this paper, the effect of convolving the Laplacian of Gaussian filter on watermarked image is shown. The Laplacian of Gaussian filter is utilized to bold the watermark information in the watermarked image. It is achieved by subtracting of the LOG filtered image from the watermarked image and adding this to the LOG filtered image. Distinction between the watermark and other parts of watermark image is augmented with the suggested process. Efficiency of proposed method is proved with doing several experiments. Two standard images; Pepper and Lena, are used for these experiments. Common image processing attacks are done on the watermark image. Robustness of the proposed method is tested by the Normalized Absolute Difference (NAD). The results show significant decrease in the NAD values. The improvement in the noise addition and blurring attacks is much better than the other attacks.

\section{References}

1. Amirgholipour KS, Naghsh-Nilchi A. A new robust digital image watermarking technique based on joint DWT-DCT transformation. 3rd International Conference on Convergence and Hybrid Information Technology ICCIT'08; Busan. 2008; 2:539-44.

2. Thanki RM, Borisagar KR. Experimental study of sparse watermarking techniques for multibiometric system. Indian Journal of Science and Technology. 2015 Jan; 8(1):42-48.

3. Amirgholipour S, Naghsh-Nilchi AR. Robust digital image watermarking based on joint DWT-DCT. JDCTA. 2009; 3(2):42-54.

4. Amirgholipour S, Mahfouzi M, Asfia M. A new pre-processing approach to improve DCT-based watermarkings extraction. International Association of IACSITSC'09; Computer Science and Information Technology-Spring Conference; Singapore. 2009. p. 131-35.

5. Amirgholipour S, Sharifi A. A pre-filtering method to improve watermark detection rate in DCT based watermarking. International Arab Journal of Information Technology. 2014; 11(2):178-85.

6. Potdar VM, Han S, Chang E. A survey of digital image watermarking techniques. 3rd IEEE International
Conference on INDIN'05, Industrial Informatics; Cambridge: UK. 2005. p. 709-16.

7. Chu WC. DCT-based image watermarking using subsampling. IEEE Transactions on Multimedia. 2003; 5(1):34-8.

8. Lin SD, Chen CF. A robust DCT-based watermarking for copyright protection. IEEE Transactions on Consumer Electronics. 2000; 46(3):415-21.

9. Sun G, Yu Y. DWT based watermarking algorithm of color images. 2nd IEEE Conference on ICIEA. Industrial Electronics and Applications; Harbin: China. 2007 May. p. 1823-26.

10. Lu W, Sun W, Lu H. Robust watermarking based on DWT and nonnegative matrix factorization. Computers and Electrical Engineering. 2009; 35(1):183-88.

11. Kutter M, Winkler S. A vision-based masking model for spread-spectrum image watermarking. IEEE Transactions on Image Processing. 2002; 11(1):16-25.

12. Levický D, Foriš P. Human visual system models in digital image watermarking. Radio Engineering. 2004; 13(4):38-43.

13. Nguyen PB, Beghdadi A, Luong M. Robust watermarking in DOG scale space using a multi-scale JND model. In Advances in Multimedia Information Processing-PCM Springer; Thailand. 2009; 5879:561-73.

14. Bouchakour M, Jeannic G, Autrusseau F. JND mask adaptation for wavelet domain watermarking. IEEE International Conference on Multimedia and Expo; Hannover. 2008. p. 201-04.

15. Li Q, Cox IJ. Using perceptual models to improve fidelity and provide resistance to volumetric scaling for quantization index modulation watermarking. IEEE Transactions on Information Forensics and Security. 2007; 2(2):127-39.

16. Pan Z, Li L, Zhang M, Zhang M. Watermark extraction by magnifying noise and applying global minimum decoder. Proceedings of Third International Conference on In Image and Graphics; Hong Kong: China. 2004. p. 349-52.

17. Alirezanejad M, Saffari V, Amirgholipour S, Sharifi AM. Effect of locations of using high boost filtering on the watermark recovery in spatial domain watermarking. Indian Journal of Science and Technology. 2014; 7(4):517-24.

18. Alirezanejad M, Amirgholipour S, Safari V, Aslani S, Arab M. Improving the Performance of Spatial Domain Image Watermarking with High Boost Filter. Indian Journal of Science and Technology. 2014 Dec; 7(12):2133-9.

19. Gonzalez RC. Digital image processing. Pearson Education India. 2009.

20. Acharya $\mathrm{T}$, Ray AK. Image processing: principles and applications. John Wiley and Sons. 2005. 